\title{
Electrophoretic Deposition of Transparent ZnO Thin Films from Highly Stabilized Colloidal Suspensions
}

\author{
M. Verde , M. Peiteado , A.C. Caballero , M. Villegas , B. Ferrari
}

Keywords:

$\mathrm{ZnO}$

Nanoflakes

Stabilization

Polyethylenimine

Electrophoretic deposition

Thin films

\begin{abstract}
A B S T R A C T
The parameters that control the stability of ZnO-nanoparticles suspensions and their deposition by electrophoretic deposition were studied, so as to organize the assembly and compaction of nanoparticles. The addition of cationic polyelectrolyte - Polyethylenimine (PEI) - with different molecular weights was investigated, in order to study their effectiveness and the influence of the molecular weight of the organic chain on suspensions dispersion. It was found that PEI with the highest molecular weight provided better dispersion conditions. Cathodic EPD was performed under previously optimized suspensions conditions and over electropolished stainless steel substrates. Experimental results showed that the EPD process in these conditions allows obtaining dense transparent $\mathrm{ZnO}$ thin films. Deposition times and intensities were optimized by analyzing the resulting thin films characteristics. Finally, the deposits were characterized by FE-SEM, AFM, and different spectroscopic techniques.
\end{abstract}

\section{Introduction}

As a wide band gap semiconductor material $\left(E_{g}=3.37 \mathrm{eV}\right), \mathrm{ZnO}$ has been extensively studied in the past. However, a renewed interest in $\mathrm{ZnO}$-based structures has recently arisen fueled by the great potential of regular arrays of $1-\mathrm{D}$ systems for a wide range of new applications [1]. ZnO thin films show unique properties, allowing the development of high speed, highly integrated, low power, and low-cost electronic and optoelectronic devices below $100 \mathrm{~nm}[2,3]$. Among the different techniques used to grow $\mathrm{ZnO}$ thin films, such as magnetron and rf magnetron sputtering, metal-organic chemical vapor deposition, and pulsed laser deposition, electrophoretic deposition (EPD) is gaining increasing interest, due to its versatility and high potential for scaling up to large volumes $[4,5]$. Actually, it might even become an alternative to the evaporative coating processes to tailor ceramic nanostructures and nanoparticles assembly.

Electrophoretic deposition is a colloidal processing method that consists in the electrically driven movement and deposition of charged particles onto a conducting substrate. Understanding the colloidal behavior of nanopowders is therefore a key point in order to prepare stable and disperse suspensions suitable for EPD [6-8]. Recently, some works focused on electrophoretic deposition of $\mathrm{ZnO}$ nanoparticles suspensions have been published discussing different colloidal aspects such as stability and particle surface charge [9-14]. This latter parameter, surface charge, is especially important for $\mathrm{ZnO}$, due to its crystalline structure. $\mathrm{ZnO}$ crystallizes in the hexagonal, non-centrosymmetric, wurtzite packing, in which planes composed of tetrahedrally coordinated $\mathrm{O}^{2-}$ and $\mathrm{Zn}^{2+}$ ions alternate, producing polar surfaces (with higher surface energy) and thus a spontaneous polarization along the $c$-axis [15]. This polarization in the (0001) direction, as well as the divergence in surface energy, is relevant to stabilization, since the presence of positively charged $(0001)-\mathrm{Zn}$ and negatively charged $(000 \overline{1})-0$ polar surfaces $[16,17]$ favors the bonding of different dispersing agents. In this work, a cationic polyelectrolyte - Polyethylenimine (PEI) - was used as dispersing agent. Positive charges in the cationic dispersant attach themselves to negative sites on the surface of the $\mathrm{ZnO}$ particles, what might result in a non-symmetric distribution.

In the present work, we discuss the results on the preparation of ZnO films by EPD starting from synthetic flake-shaped nanoparticles. Some of the critical parameters related to the preparation of aqueous suspensions of these nanopowders, specifically solid contents and addition of a conventional cationic polyelectrolyte, were already studied in a previous work [18]. The interrelation between these parameters has a major influence on the deposits morphology. In this study, influence of the molecular weight of that cationic electrolyte - Polyethylenimine - (used to give the $\mathrm{ZnO}$ particles a positive charge potential and good dispersion in aqueous media) is analyzed. Later on, electrophoretic arrangement and film formation are discussed. 


\section{Experimental procedure}

$\mathrm{ZnO}$ nanoparticles were synthesized by direct precipitation. An aqueous solution of zinc acetate $\left(\mathrm{Zn}\left(\mathrm{CH}_{3} \mathrm{COO}\right)_{2} \cdots 2 \mathrm{H}_{2} \mathrm{O}\right)$ (Sigma Aldrich (o., USA) was prepared, and precipitation was carried out at $\mathrm{pH}>8$ by the addition of $\mathrm{NaOH} 1 \mathrm{M}$. The resulting precipitate was washed and filtered. A thermal treatment at $200^{\circ} \mathrm{C}$ for $3 \mathrm{~min}$ was enough to decompose the remaining starting reagents and to obtain $\mathrm{ZnO}$ nanopowders. Further synthesis details have been previously reported in the literature [19]. Complete morphological characterization of $\mathrm{ZnO}$ powders was carried out: specific surface area was measured by $\mathrm{N}_{2}$ adsorption - BET method (Monosorb Surface Area Analyser MS-13, Quantachrome Co., USA) and density $(\rho)$ by helium pycnometry (Multipycnometer, Quantachrome Co., USA). Particle size and morphology were examined by cold field emission scanning electron microscopy (FE-SEM) in an S-4700 microscope (Hitachi, JAPAN), by transmission electron microscopy (TEM), using a H-7100 model (Hitachi, Japan) and by high-resolution transmission electron microscopy (HRTEM) in a JEOL JEM 2011 (Jeol Ltd., Japan). Crystalline phase was characterized by Xray diffraction (XRD) (D8 Advance, Bruker, Germany), using $\mathrm{Cu}$ $K \alpha$ radiation.

Suspensions dispersion and stability were evaluated in terms of particle size distribution and zeta potential. Both parameters were measured by dynamic light scattering (DLS) and laser Doppler velocimetry, respectively, in a Zetasizer Nano ZS (Malvern S, UK). Mechanical stirring and sonication (Ultrasonication Probe, UP 400S, Hielscher, Germany) were used as dispersing methods, in order to break $\mathrm{ZnO}$ agglomerates, and $\mathrm{pH}$ and addition of cationic polyelectrolytes - Polyethylenimines - with different molecular weights (PEI, Sigma Aldrich Co., USA) - were optimized so as to prevent re-agglomeration.pH was adjusted by the addition of small amounts of $0.1 \mathrm{M} \mathrm{HCl}$ or $\mathrm{KOH}$ and controlled by a standard glass electrode $\mathrm{pH}$ meter (Metrohm AG, Germany). Influence of the molecular weight of the cationic dispersant was also evaluated.

The protocol for the preparation of the suspensions is described as follows: addition of the corresponding Polyethylenimine to distilled water, addition of the $\mathrm{ZnO}$ powder, $\mathrm{pH}$ adjustment to 7-8 regarding $\mathrm{ZnO}$ dissolution at $\mathrm{pH}<6$ [20] - and finally sonication. For particle size measurements, these suspensions were diluted over distilled water to an adequate concentration $(\leqslant 0.01 \mathrm{~g} / \mathrm{l}), \mathrm{pH}$ was readjusted, and $15 \mathrm{~s}$ of sonication was applied. In order to check that the medium $\mathrm{pH}$ was not acid and prevent $\mathrm{ZnO}$ dissolution, $\mathrm{pH}$ was always measured after sonication, obtaining values $\geqslant 7$.

ZnO films were shaped by EPD on commercial steel substrates (AISI 304) of $50 \times 25 \times 0.5 \mathrm{~mm}$ that were previously electropolished. Electropolishing was carried out using an electrolyte containing perchloric acid, ethanol and distilled water and applying $38 \mathrm{~V}$ for $1 \mathrm{~min}$ (Electromet 4, Buehler, USA). The counter electrode was a platinum foil of similar dimensions, separated from the work electrode by a distance of $2 \mathrm{~cm}$ in the electrophoretic cell. EPD was performed under galvanostatic conditions using a high voltage power source (2611 System SourceMeter, Keithley Instruments Inc., USA), applying current densities between $9.3 \times 10^{-3}$ and $0.2 \mathrm{~mA} / \mathrm{cm}^{2}$ and deposition times from 1 to $10 \mathrm{~min}$. Potential drop between the electrodes never exceeds $5 \mathrm{~V}$ in order to avoid $\mathrm{H}_{2}$ bubbling (due to water electrolysis). After EPD, the samples were left to dry at room conditions.

The obtained films were microstructurally characterized by Cold Field Emission Scanning Electron Microscopy (FE-SEM), with Energy Dispersive Spectroscopy Microanalysis (EDS), and by Atomic Force Microscopy (AFM). AFM measurements were carried out with a Cervantes AFM System (Nanotec Electronica S.L., SPAIN) operating in non-contact dynamic mode with amplitude modulation and non-coated silicon nitride cantilevers.

\section{Results and discussion}

\subsection{Powder characteristics}

The measured specific surface area and density of powders were $140 \mathrm{~m}^{2} / \mathrm{g}$ and $4.36 \mathrm{~g} / \mathrm{cm}^{3}$ respectively, whereas $\mathrm{ZnO}$ nanoparticles diameter obtained from the BET approximation is $9.8 \mathrm{~nm}$. In the SEM micrograph of the as-prepared powder shown in Fig. 1a, a homogeneous population of flake-shaped particles, with thickness in the order of nm, can be observed. Particle size can be confirmed in the high-resolution (HR-TEM) micrograph in Fig. 1b. As it can be observed, the width of the flakes along the ab plane in the micrograph ranges from 15 to $30 \mathrm{~nm}$.

The calculated equivalent spherical diameter of $\mathrm{ZnO}$ particles $\left(d_{\mathrm{BET}}\right)$ is lower than the primary particle size measured by TEM because of the high shape factor of the nano-flakes (around 10). Furthermore, the lattice fringes corresponding to the $(10 \overline{1} 0)$ plane of the hexagonal flake can be seen in the HRTEM micrograph. These correspond to the basal plane of the $\mathrm{ZnO}$ flake-like particle, which is parallel to the surface and perpendicular to the (0001) polarized plane. A view over SEM and TEM images evidences the high shape factor of the samples and their large agglomeration state.

XRD diffractogram of the powder after calcination (Fig. 2) shows that the thermal treatment of $3 \mathrm{~min}$ at $200^{\circ} \mathrm{C}$ was enough to decompose all rests of the starting products, as it presents a small background signal and narrow peaks which correspond only to $\mathrm{ZnO}$. The inset in the figure shows the lattice parameters estimated using the FullProf $2 \mathrm{k}$ program [21].

\subsection{Suspension preparation and stabilization}

Dispersing conditions were optimized following previously reported ones [18] so as to obtain the best results regarding these parameters. Nano-ZnO suspensions were prepared with $0.1 \mathrm{~g} / \mathrm{l}$ solid contents and dispersed by mechanical stirring, adjusting the aqueous medium to different $\mathrm{pH}$, and applying high power

\section{(a)}
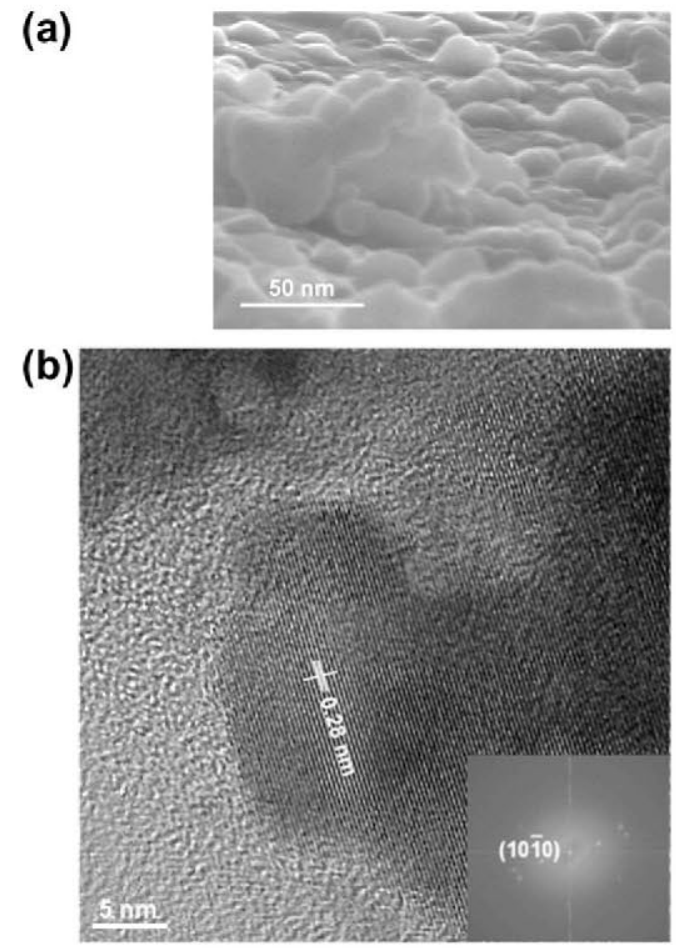

Fig. 1. (a) SEM and (b) HRTEM micrographs of the synthesized ZnO nanopowder. 


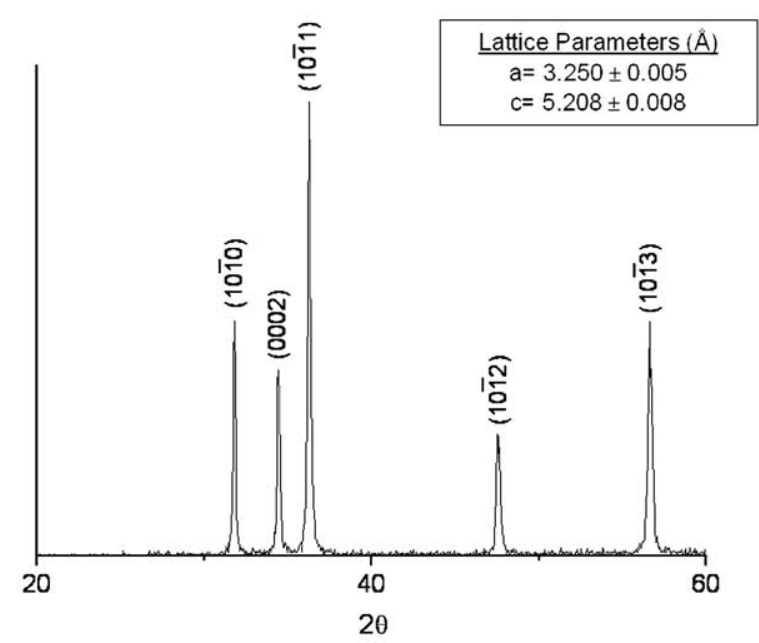

Fig. 2. XRD pattern of the synthesized powder (after thermal treatment of $200{ }^{\circ} \mathrm{C}$ for $3 \mathrm{~min}$ ). ZnO (JCPDS No. 01-079-0206). Calculated lattice parameters are shown in the inset.

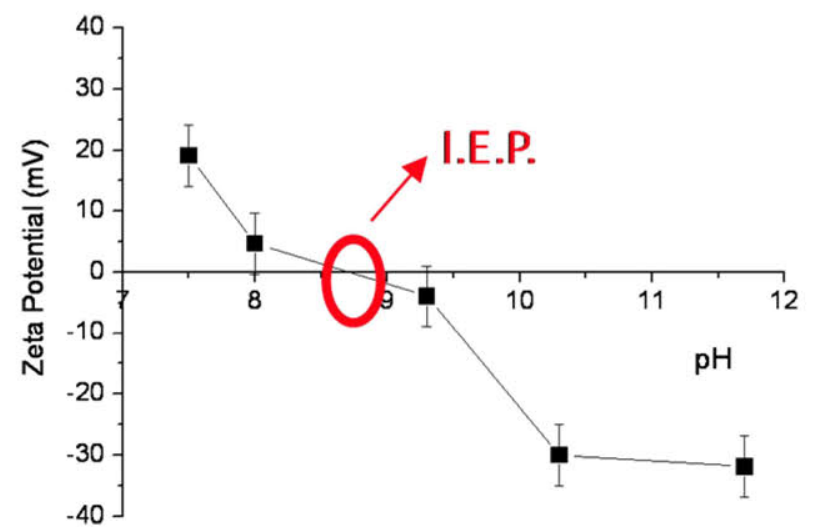

Fig. 3. Zeta potential vs pH measurements of a $0.1 \mathrm{~g} / \mathrm{l} \mathrm{ZnO}$ suspension in $\mathrm{KCl} 10^{-2} \mathrm{~N}$ with $30 \mathrm{~s}$ sonication. The experimental isoelectric point (IEP) is signaled in red. (For interpretation of the references to color in this figure legend, the reader is referred to the web version of this article.)

ultrasonication. First of all, superficial behavior of the powder when suspended in an aqueous media was characterized by zeta potential measurements (Fig. 3) as a function of $\mathrm{pH}$, and it was observed that, as inferred from the $\mathrm{XRD}$, the synthesized $\mathrm{ZnO}$ nanopowder has no impurities on its surface, which affect its
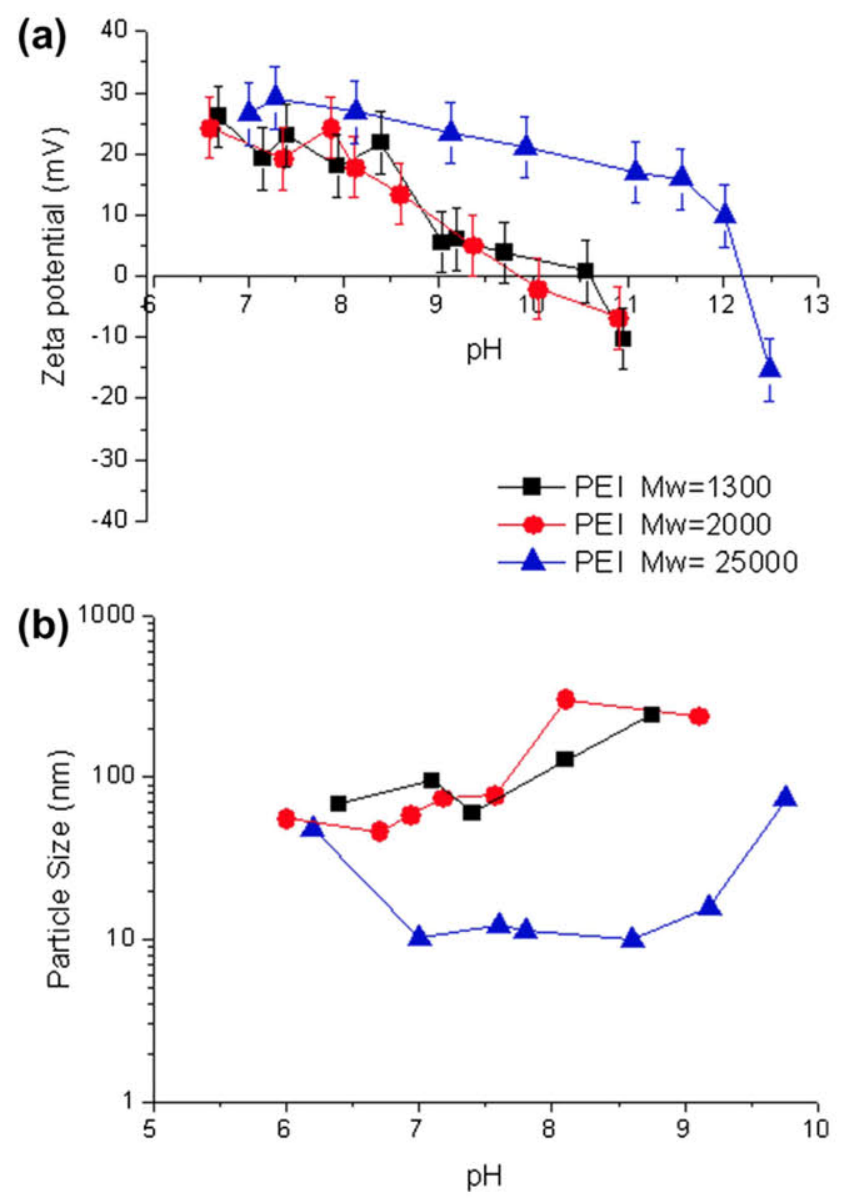

Fig. 4. (a) Zeta potential and (b) particle size variation as a function of Polyethylenimines molecular weight vs $\mathrm{pH}$.

stabilization, since the measured isoelectric point is in agreement with those reported in the literature, ranging from 8.5 to 10.3 $[22,23]$. In order to carry on their cathodic EPD, ZnO nanoflakes should be positively charged, thus Polyethylenimine (PEI) - a cationic polyelectrolyte - was chosen to modify particles surface, improving and extending their dispersion and stabilization in aqueous suspension. Structurally, PEI has a high positive charge, due to the fact that after every two carbon atoms, there is a secondary amino nitrogen atom able to protonate itself (Table 1) [24].

PEI maintains its protonated structure even at basic $\mathrm{pH}$, being an effective dispersant in a broad $\mathrm{pH}$ range. Dispersing capability

Table 1

PEI formula and structure.

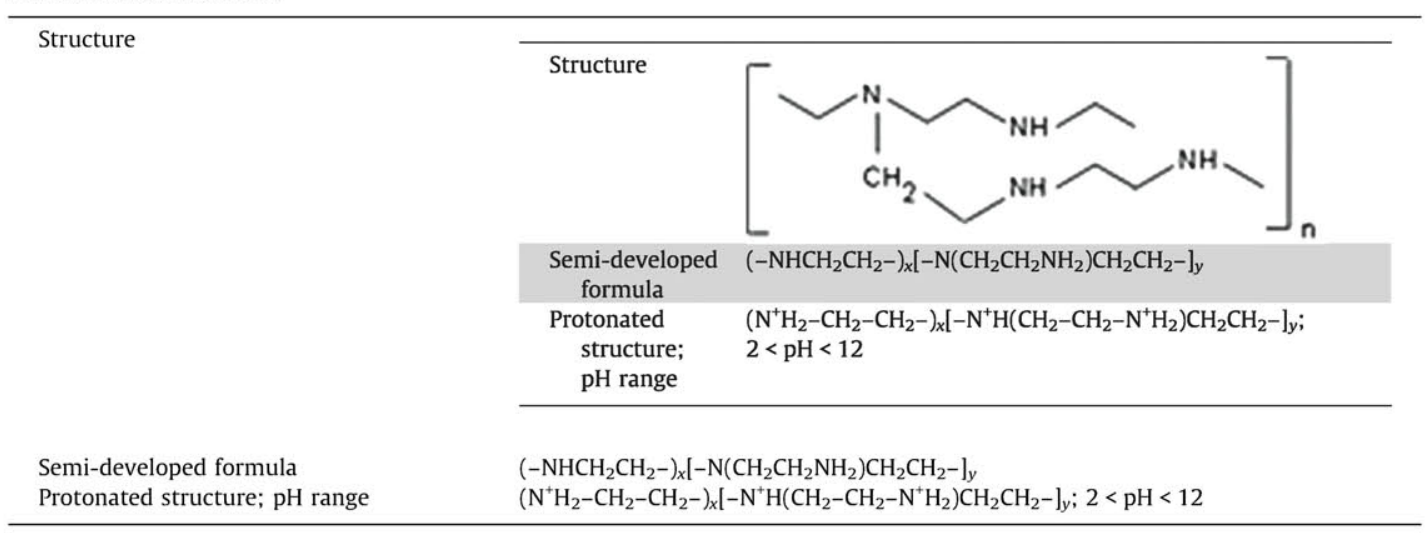


of PEI with $\mathrm{Mw}=2000$ was preliminary discussed in a previous work [18]. However, in this work, a thorough study of Polyethylenimines with different molecular weights $(\mathrm{Mw}=1300,2000$ and 25,000 ) has been carried out, so as to determine the effect of the latter on dispersion by means of zeta potential and particle size versus $\mathrm{pH}$ measurements (Fig. 4). For this purpose, suspensions were prepared with $1 \mathrm{~g} / \mathrm{l}$ solids content, $1 \mathrm{wt} \%$ PEI and $15 \mathrm{~s}$ of sonication in order to break soft agglomerates.

By following this procedure, and according to previous zeta potential measurements (Fig. 3 ), in the chosen $\mathrm{pH}$ range $(7<\mathrm{pH}<8$ ), $\mathrm{ZnO}$ particles have a slightly positive surface charge and thus certain number of negative sites on their surface. Therefore, adsorption sites on the particles surface and charge of the organic chain are always the same. However, the number of polymeric chains that adsorb onto the particles surface depends on the size of the organic chain. The value of $1 \mathrm{wt} \%$ PEI was previously optimized [18] by studying the $\zeta$-potential vs wt.\% PEI saturation curve, which enables the estimation of the adsorbed amount of dispersant [25]. In this way, we can expect that the percentage of adsorbed PEI will be smaller for the one with higher molecular weight and higher for the one with smaller molecular weight. Attending to the low concentration of the suspensions, the excess of heavier PEI can be neglected. In fact, besides the stability of the suspension, the key parameter of the electrophoretic process is the electrophoretic mobility, directly proportional to the zeta potential, which depends on the protonation of the PEI at the working $\mathrm{pH}$ value once it is anchored to the particle surface. In this way, results showed that Polyethylenimine with the highest molecular weight $(\mathrm{Mw}=$ 25,000 ) provides a greater repulsive electrostatic interaction, shifting the isoelectric point to $\mathrm{pH}>12$ (Fig. 4a). PEI adsorption and effect is also clear in the equivalent-sphere size distribution measurement versus pH values shown in Fig. 4b. The electro-steric effect favored by the highest molecular weight PEI maintains $\mathrm{ZnO}$ nanoparticles dispersion and prevents re-agglomeration, keeping the agglomerates size below $100 \mathrm{~nm}$ after sonication. Such low size values are due to the fact that the DLS technique measures the hydrodynamic diameter using the MIE theory, which considers spherical particles and overlooks the high shape factor of the $\mathrm{ZnO}$ nanoflakes.

In view of the experimental results, the Polyethylenimine chosen to prepare suspensions for EPD was the one with $\mathrm{Mw}=$ 25,000 , since it allows working in the chosen $\mathrm{pH}$ range, $7<\mathrm{pH}<$ 8 , providing the optimum zeta potential and particle size values. Attending to the surface structure of the $\mathrm{ZnO}$ nanoparticles, a
Table 2

ZnO EPD suspensions conditions.

\begin{tabular}{llllll}
\hline Powder/medium & $\mathrm{Cc}(\mathrm{g} / \mathrm{l})$ & Dispersant & $\mathrm{pH}$ & $d_{\mathrm{v} 50}(\mathrm{~nm})$ & $\zeta(\mathrm{mV})$ \\
\hline $\mathrm{ZnO} / \mathrm{H}_{2} \mathrm{O}$ & $0.1-1$ & $\begin{array}{l}\mathrm{PEl}(\mathrm{Mw}=25,000) \\
1 \mathrm{wt} \%\end{array}$ & $7-8$ & 10 & $>25$ \\
& & & & \\
\hline
\end{tabular}

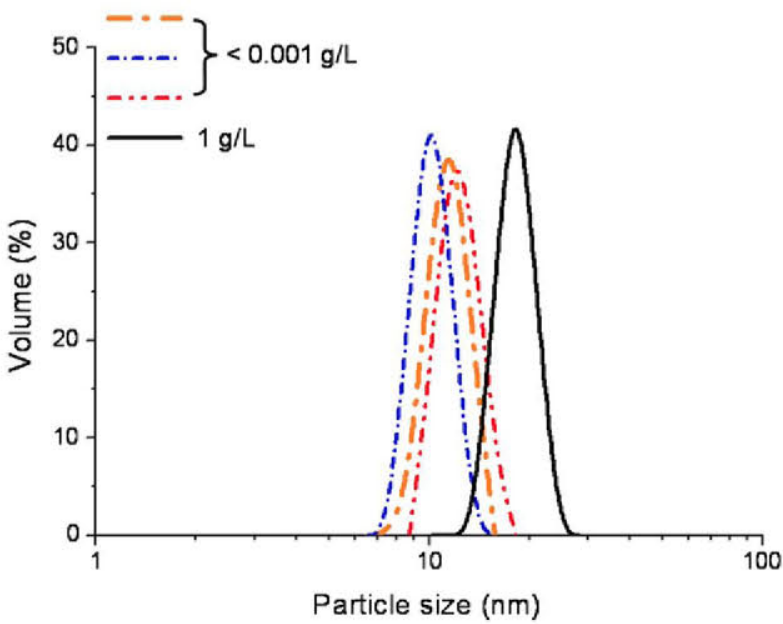

Fig. 6. Particle size measurements as a function of solid contents: $<0.01 \mathrm{~g} / \mathrm{l}$ and $1 \mathrm{~g} / \mathrm{l}$ suspensions, both with $1 \% \mathrm{PEl}(\mathrm{Mw}=25,000)$ and sonication. Dispersion technique is effective in all the solid contents range.

non-symmetric "jellyfish-like" structure should be figured, in which positive charges in the Polyethylenimine mainly attach to negative sites on the (000 $\overline{1})-O$ polar surface of the $\mathrm{ZnO}$ particles (Fig. 5). This way dispersion of $\mathrm{ZnO}$ nanoparticles is enhanced by the bigger size of the chain of PEI with $\mathrm{Mw}=25,000$, due to both electrostatic and steric effects. Moreover, during electrophoresis, the "jellyfish" structure might favor the motion and subsequent deposition of the nanoflakes in the direction parallel to the substrate.

Under these optimized conditions (summarized in Table 2), suspensions are stable and $\mathrm{ZnO}$ particles surface is positively charged, which favors their cathodic deposition by electrophoresis. Particle size measurements remain similar in the concentration range from 0.1 to $1 \mathrm{~g} / \mathrm{l}$, in which particles arrange themselves parallel to the substrate during deposition [18]. Fig. 6 shows how the dispersion

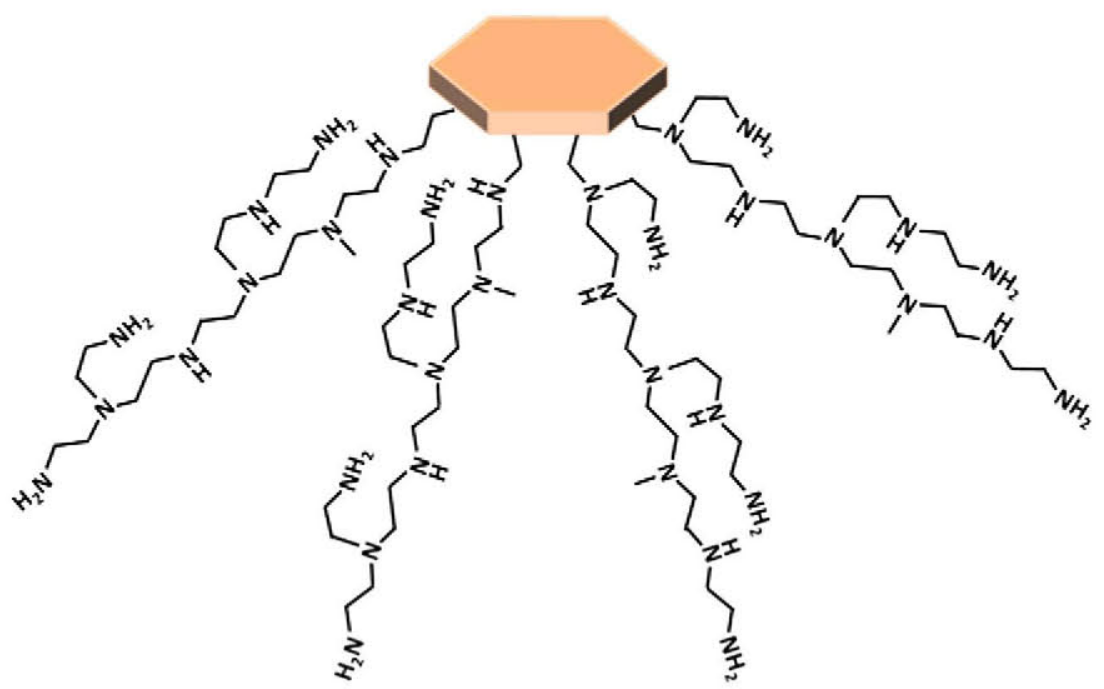

Fig. 5. "Jellyfish-like" structure of $\mathrm{ZnO}$ with PEI attached to the negative surface. 


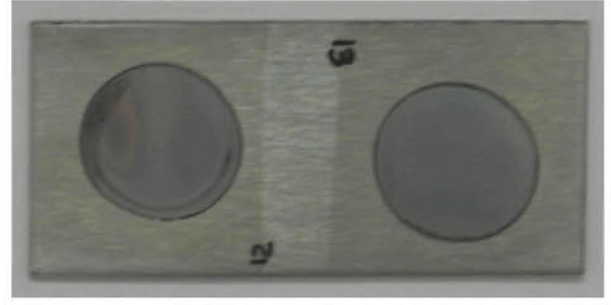

Fig. 7. Optical photograph of an electropolished substrate coated with a $\mathrm{ZnO}$ film from a $1 \mathrm{~g} / 1$ suspension.

technique is effective in this range, measuring particle sizes $\left(d_{\mathrm{v} 50}\right)$ around $20 \mathrm{~nm}$ for $1 \mathrm{~g} / 1$ suspensions.

\subsection{ZnO films obtained by EPD}

EPD was performed under galvanostatic conditions, using 0.1 and $1 \mathrm{~g} / \mathrm{l} \mathrm{ZnO}$ aqueous suspensions, with $1 \mathrm{wt} . \% \mathrm{PEI}$ and applying the optimized parameters for particles dispersion and stabilization described above. Low current densities, ranging from $9.3 \times 10^{-3}$ to $0.2 \mathrm{~mA} / \mathrm{cm}^{2}$, were applied to nano-ZnO suspensions in order to avoid $\mathrm{H}_{2}$ bubbling (due to water electrolysis). The deposition rates of $0.1 \mathrm{~g} / 1$ suspensions were of $0.04 \mathrm{mg} / \mathrm{cm}^{2} \cdot \mathrm{min}$ for PEI with $\mathrm{Mw}=2000$ and of $0.003 \mathrm{mg} / \mathrm{cm}^{2} \cdot \mathrm{min}$ for PEI with $\mathrm{Mw}=25,000$.

A general view of a stainless steel substrate with two electropolished and $\mathrm{ZnO}$-coated areas is shown in Fig. 7. As it can be observed, the $\mathrm{ZnO}$ films from $1 \mathrm{~g} / \mathrm{l}$ suspensions were translucent. However, $\mathrm{ZnO}$ films from $0.1 \mathrm{~g} / \mathrm{l}$ suspensions were transparent (non-detectable by visual inspection). This transparency points out the high packing density of the particles in the film [11], as will later be confirmed by SEM. Fig. 8a) shows an optical micrograph of the $1 \mathrm{~g} / \mathrm{l}$ coated substrate in Fig. 7. From left to right, three different areas can be distinguished: (i) non-electropolished - non-coated area, (ii) non-electropolished - coated area, and (iii) electropolished and coated area. FE-SEM micrographs of the electropolished and coated area (iii) show a highly dense nanostructured film of $\mathrm{ZnO}$ particles with $\mathrm{ZnO}$ clusters dispersed on top (Fig. 8b-d).

FE-SEM micrographs of thin films obtained from $0.1 \mathrm{~g} / 1$ suspensions show a dense $\mathrm{ZnO}$ structure that presents disperse pores due to the deposition process - on its surface (Fig. 9a). However, this porosity does not affect the films transparency, since the pore size is below $50 \mathrm{~nm}$. A FE-SEM micrograph of the same film after a mild thermal treatment $\left(300^{\circ} \mathrm{C} / 2 \mathrm{~h}\right)$ shows a slight decrease in this

\section{(a)}

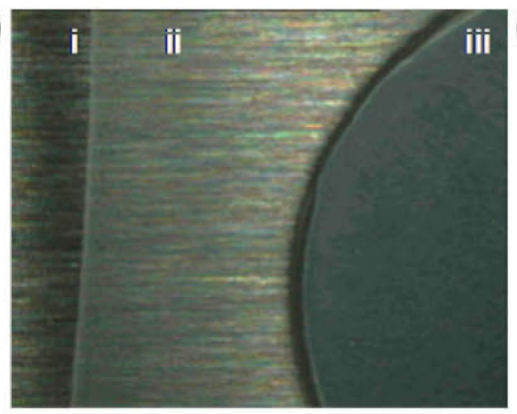

(c)

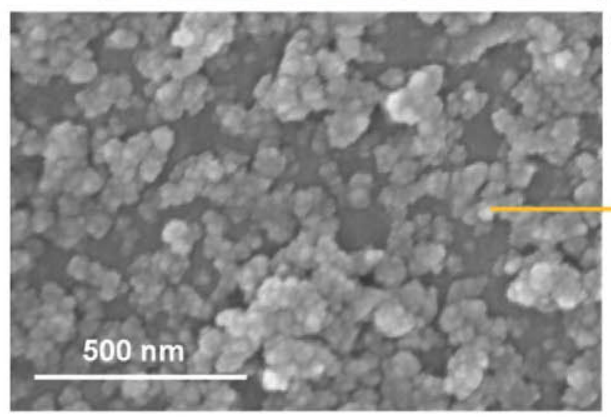

(b)

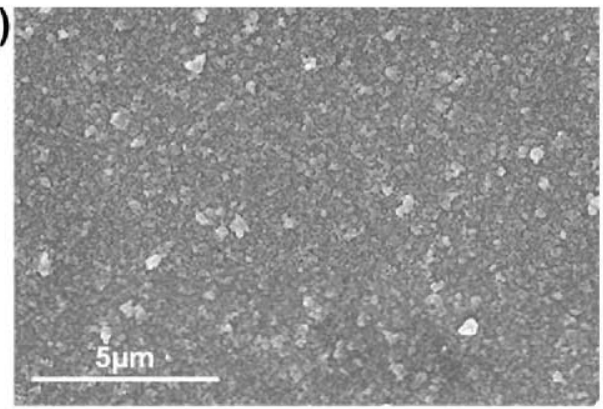

(d)

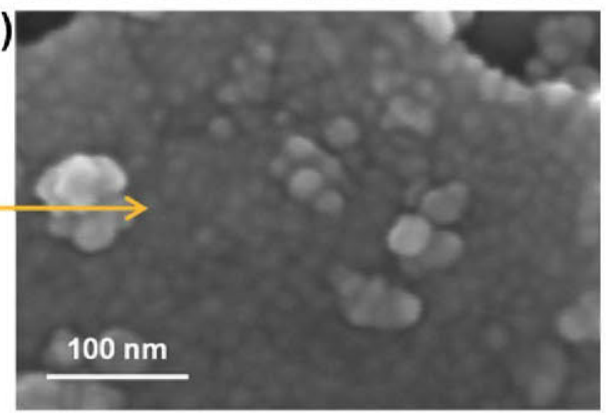

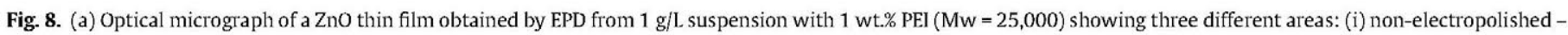

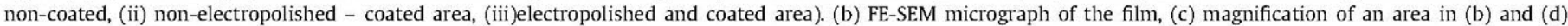
magnification of an area in (c).

\section{(a)}

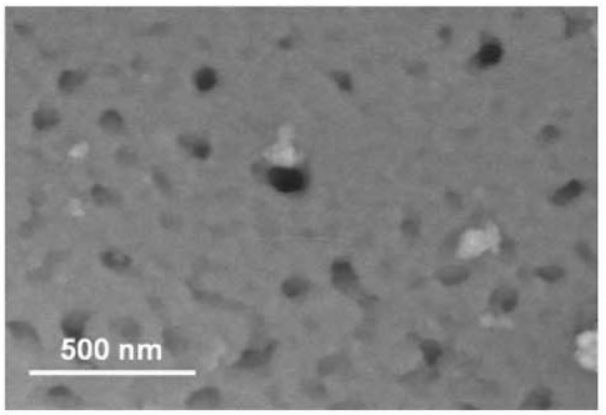

(b)

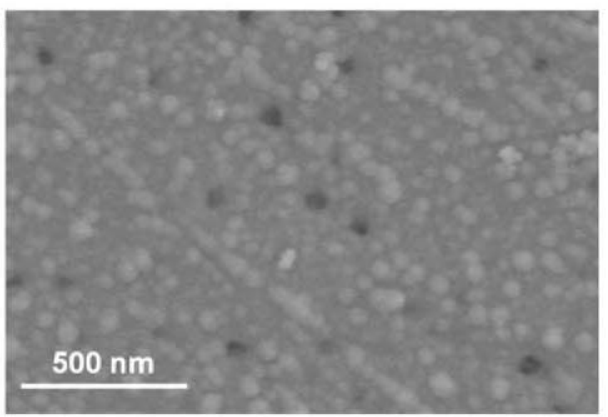

Fig. 9. (a) FE-SEM micrograph of a ZnO thin film obtained by EPD from $0.1 \mathrm{~g} / 1$ suspension with $1 \mathrm{wt} \%$ PEI $(\mathrm{Mw}=25,000)$. (b) FE-SEM micrograph of the film after a mild thermal treatment $\left(300^{\circ}-2 \mathrm{~h}\right)$ 

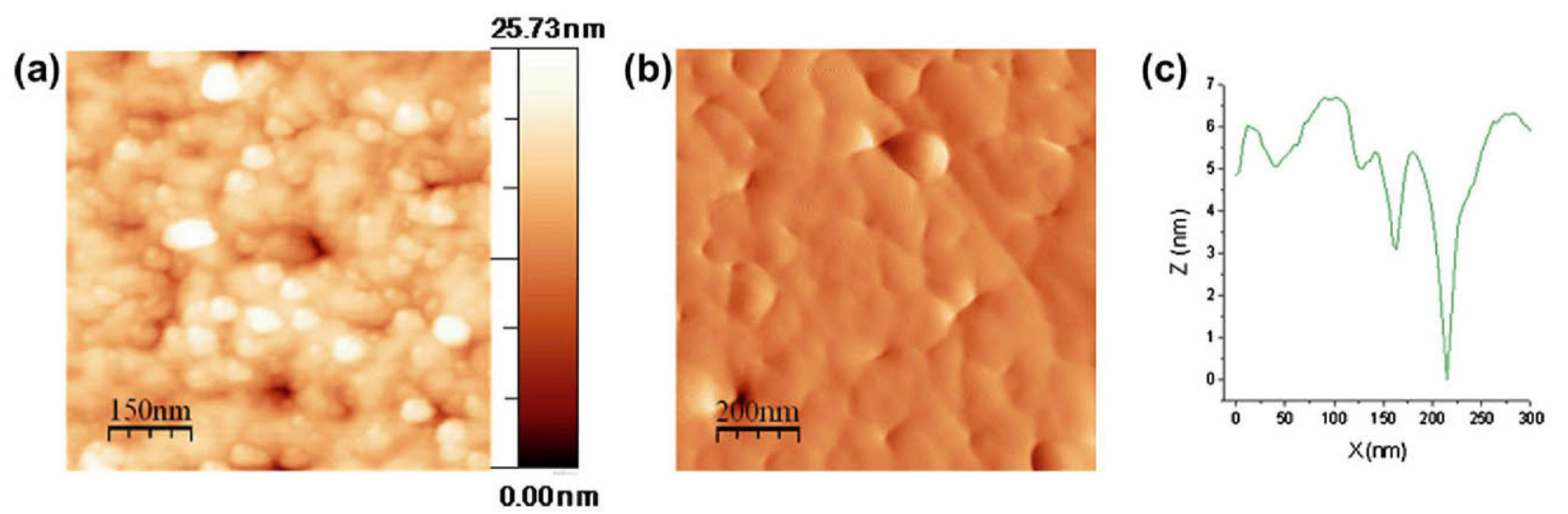

Fig. 10. AFM micrographs of ZnO thin films deposited from $0.1 \mathrm{~g} / \mathrm{l}$ suspensions; (a) surface topography; (b) amplitude map; (c) profile of the topography in (a).

porosity and "rows" of aligned particles, evidencing the beginning of interparticle necks growth (Fig. 9b).

Homogeneous films were obtained for all applied electric fields and suspensions, independently of the concentration. The high packing density of the films is due to the morphology and orientation of the particles. Nanoparticles arrangement is usually determined by the electrode surrounding conditions, i.e., ionic gradients [26], dielectric breakdown of a non-conducive template [27], the electro hydrodynamic of the fluid [28], increase in solid contents[29]. In this case, during electrophoretic movement and due to the addition of the cationic dispersant resulting in the
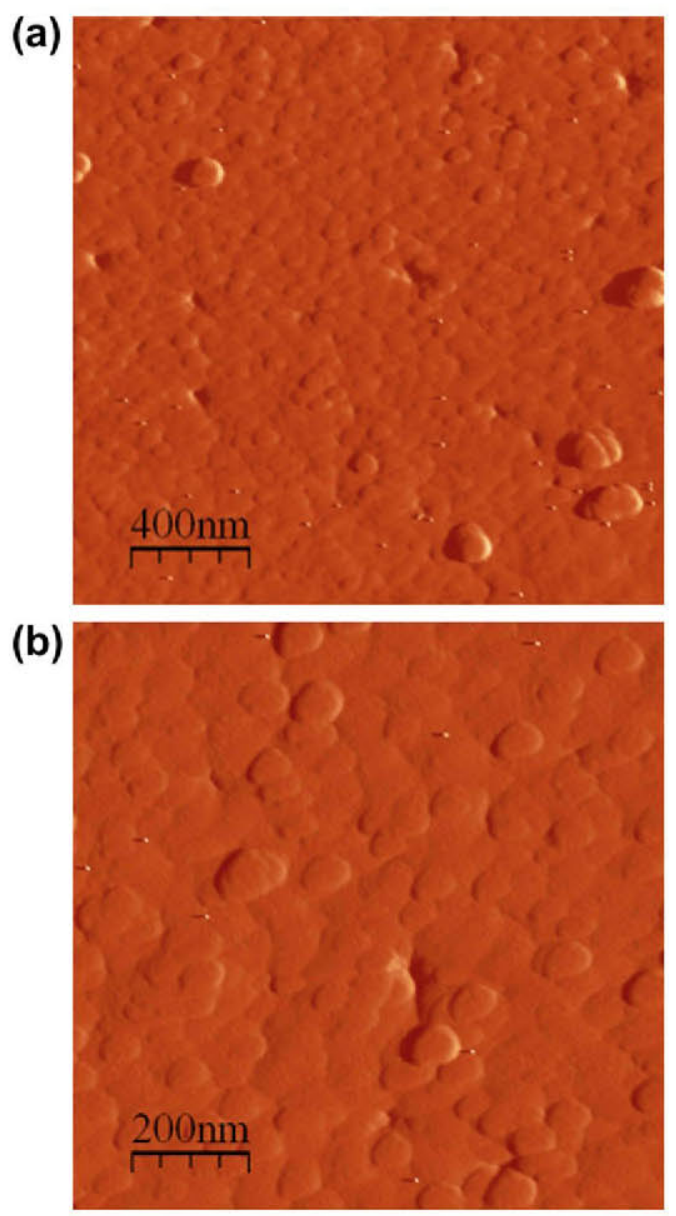

Fig. 11. AFM amplitude maps of the $\mathrm{ZnO}$ thin film deposited from $0.1 \mathrm{~g} / \mathrm{l}$ suspension shown in Fig. 9a. jellyfish-like structure, orientation of the majority of the particles is opposite to that favored by hydrodynamics, i.e., $\mathrm{ZnO}$ nanoflakes with PEI attached to their surface move with their basal plane parallel to the substrate. However, when reaching the substrates surface, PEI, which is a weak cationic polyelectrolyte, loses charge and reduces its electrostatic effect as dispersant due to the high $\mathrm{pH}$ increase near the cathode surface [30,31]. However, when $\mathrm{ZnO}$ nanoflakes arrive on the substrates surface, the steric effect of PEI helps them organize in the same way they moved, aligning their principal dimension parallel to the substrate, according to the electrohydrodynamics and forming clusters of 50-100 $\mathrm{nm}$ which appear to be the building blocks of the structure. Besides the effect of PEI and hydrodynamic forces, formation of clusters is also determined by $\mathrm{ZnO}$ characteristics: $\mathrm{ZnO}$ particles arrange themselves in accordance with the polarity of their surface $[17,32,33]$ and with their intrinsic anisotropy in the presence of an electric field (since they exhibit piezoelectric effect) [34].

Films deposited from $0.1 \mathrm{~g} / \mathrm{l}$ suspensions were finally studied by atomic force microscopy in order to verify the topology of the surface and confirm the orientation observed by SEM. Fig. 10a shows a 2D AFM topography of one of these films, in which it can be observed that the nanoparticles diameter in the film is of 20$40 \mathrm{~nm}$. The amplitude map (Fig. 10b) shows the organization of the platelets parallel to the substrate. Moreover, the profile of the topography in Fig. 10a shows a local roughness $<10 \mathrm{~nm}$, which confirms the orientation of the particles parallel to the substrate, as it corresponds to approximately one, two, or three times the thickness of the nanoflakes (Fig. 10c).

Results of the scanning of a small area of the film in Fig. 9a are shown in Fig. 11a and b. The full topography of the film can be obtained in an accurate AFM scanning by decreasing the amplitude of the resonance movement of the cantilever and increasing the gain. $\mathrm{ZnO}$ arrays corresponding in size with the structure shown in FESEM (Fig. 9a) can be observed in the amplitude maps in Fig. 11. All those images evidence the ordered deposition of $\mathrm{ZnO}$ flake-like nanoparticles onto the electropolished stainless steel surface which is promoted by two main facts: one, the low solid contents and high stability of the suspensions; and two, the one-side steric effect provided by adsorption of the heavier PEI on one of the particles surface during both electrophoresis and deposition.

\section{Conclusions}

The optimum conditions to prepare films exhibiting an extremely packed structure before sintering through the oriented compaction of flake-like nanoparticles of $\mathrm{ZnO}$ have been described.

Surface of flake-shaped $\mathrm{ZnO}$ nanoparticles was modified by addition of a cationic polyelectrolyte - Polyethylenimine (PEI). It 
was found that the PEI with the highest molecular weight provides larger electrostatic repulsive interaction between nanoparticles and a larger steric effect, and this is stable suspensions. Moreover, the jellyfish-like structure formed with the high molecular weight PEI favors hydrodynamic forces that align Zno flake-like nanoparticles parallel to the surface of the substrates during EPD. This procedure allowed obtaining of $\mathrm{ZnO}$ thin films with extremely interesting features such as high packing green density and room transparency even previously to thermal treatment.

\section{Acknowledgments}

The authors gratefully acknowledge financial support from the Spanish Science and Innovation Ministry (MCI) through the Projects MAT2009-14448-C02-01, MAT2010-16614, IPT-3100002010-0012, and IPT-120000-2010-33 (Spain). M. Verde acknowledges the CSIC for the concession of a Jae-Predoc 2009 fellowship.

\section{References}

[1] C. Klingshirn, J. Fallert, H. Zhou, J. Sartor, C. Thiele, F. Maier-Flaig, D. Schneider, H. Kalt, Phys. Status Solidi B 247 (2010) 1424-1447.

[2] H.E. Ruda, J.C. Polanyi, J. Yang, Z. Wu, U. Philipose, T. Xu, S. Yang, K.L. Kavanagh, J.Q. Liu, L. Yang, Y. Wang, K. Robbie, J. Yang, K. Kaminska, D.G. Cooke, F.A. Hegmann, A.J. Budz, H.K. Haugen, Nanoscale Res. Lett. 1 (2006) 99-119.

[3] J. Xiong, S.N. Das, B. Shin, J.P. Kar, J.H. Choi, J.-M. Myoung, J. Colloid Interface Sci. 350 (2010) 344-347.

[4] I. Corni, M.P. Ryan, A.R. Boccaccini, J. Eur. Ceram. Soc. 28 (2008) 1353-1367.

[5] A.R. Boccaccini, J.A. Roether, B.J.C. Thomas, M.S.P. Shaffer, E. Chavez, E. Stoll, E.J. Minay, J. Ceram. Soc. Jpn. 114 (2006) 1-14.

[6] K. Wu, I. Zhitomirsky, Int. J. Appl. Ceram. Technol. 8 (2010) 920-927.

[7] P. Sarkar, P.S. Nicholson, J. Am. Ceram. Soc. 79 (1996) 1987-2002.

[8] O.O. Van Der Biest, L.J. Vandeperre, Annu. Rev. Mater. Sci. (1999) 327-352.
[9] K. Govender, D.S. Boyle, P. O'Brien, Proc. - Electrochem. Soc. PV 2003-32 (2006) 114-128.

[10] P. Lommens, D. Van Thourhout, P.F. Smet, D. Poelman, Z. Hens, Nanotechnology 19 (2008) 245-301.

[11] Y. Wang, I.-C. Leu, M.-H. Hon, J. Am. Ceram. Soc. 87 (2004) 84-88.

[12] L. Miao, S. Cai, Z. Xiao, J. Alloys Compd. 490 (2010) $422-426$.

[13] F. Tang, Y. Sakka, T. Uchikoshi, Mater. Res. Bull. 38 (2003) 207-212.

[14] F. Tang, T. Uchikoshi, Y. Sakka, J. Am. Ceram. Soc. 85 (2002) 2161-2165

[15] C. Wöll, Prog. Surf. Sci. 82 (2007) 55-120.

[16] Z.L. Wang, Mater. Today 7 (2004) 26-33.

[17] M. Peiteado, T. Jardiel, F. Rubio, A.C. Caballero, Mater. Res. Bull. 45 (2010) 1586-1592.

[18] M. Verde, A.C. Caballero, Y. Iglesias, M. Villegas, B. Ferrari, J. Electrochem. Soc. 157 (2010) H55-H59.

[19] J.E. Rodriguez-Paez, A.C. Caballero, M. Villegas, C. Moure, P. Duran, J.F. Fernandez, J. Eur. Ceram. Soc. 21 (2001) 925-930.

[20] A. Degen, M. Kosec, J. Eur. Ceram. Soc. 20 (2000) 667-673.

[21] J. Rodríguez-Carvajal, Physica B 192 (1993) 55-69.

[22] J.S. Reed, Introduction to the Principles of Ceramic Processing, John Wiley \& Sons, New York, 1986.

[23] G.A. Parks, Chem. Rev. 65 (1965) 177-198.

[24] E.M. Kosacheva, D.B. Kudryavtsev, R.F. Bakeeva, A.I. Kuklin, A.K. Islamov, L.A. Kudryavtseva, V.F. Sopin, A.I. Konovalov, Colloid J. 68 (2006) 713-720.

[25] M.E. Helgeson, T.K. Hodgdon, E.W. Kaler, N.J. Wagner, M. Vethamuthu, K.P. Ananthapadmanabhan, Langmuir 26 (2010) 8049-8060.

[26] L. Stappers, L. Zhang, O. Van, J. der Biest, J. Fransaer, J. Colloid Interface Sci. 328 (2008) 436-446.

[27] J.-H. Lee, I.-C. Leu, Y.-W. Chung, M.-H. Hon, Nanotechnology 17 (2006) 44454450.

[28] Y. Solomentsev, M. Bahmer, J.L. Anderson, Langmuir 13 (1997) 6058-6061.

[29] L. Besra, M. Liu, Prog. Mater. Sci. 52 (2007) 1-61.

[30] L. Besra, T. Uchikoshi, T.S. Suzuki, Y. Sakka, J. Eur. Ceram. Soc. 30 (2010) $1187-$ 1193.

[31] M. Mishra, S. Bhattacharjee, L. Besra, H.S. Sharma, T. Uchikoshi, Y. Sakka, J. Eur. Ceram. Soc. 30 (2010) 2467-2473.

[32] R.W. Nosker, P. Mark, J.D. Levine, Surf. Sci. 19 (1970) 291-317.

[33] U. Diebold, S.-C. Li, M. Schmid, Annu. Rev. Phys. Chem. (2010) 129-148.

[34] U. Özgur, J. Appl. Phys. 98 (2005) 1-103. 